PHYSICAL REVIEW E 94, 019903(E) (2016)

\title{
Publisher's Note: Eigenvector dynamics under perturbation of modular networks [Phys. Rev. E 93, 062312 (2016)]
}

Somwrita Sarkar, Sanjay Chawla, P. A. Robinson, and Santo Fortunato

(Received 5 July 2016; published 11 July 2016)

DOI: 10.1103/PhysRevE.94.019903

This paper was published online on 20 June 2016 with the omission of a second affiliation for the second author. The affiliations for Sanjay Chawla should read as Qatar Computing Research Institute, Hamad Bin Khalifa University (HBKU), Qatar Foundation, Doha, Qatar and Faculty of Engineering and IT, University of Sydney, Australia NSW 2006. The paper has been corrected as of 30 June 2016. The text is correct in the printed version of the journal. 